\title{
ELECTRICAL ENGINEERING EDUCATIONAL SOFTWARE BASED ON MATLAB AND ITS GRAPHIC USER INTERFACE (GUI)
}

\author{
Nani Fadzlina Naim ${ }^{1 \star}$, Muhammad Fauzi Khairul Fisal ${ }^{2}$, Suzi Seroja Sarnin ${ }^{3}$, \\ Norsuzila Ya'acob4 \\ ${ }^{1}$ Dr., Fakulti Kejuruteraan Elektrik, Universiti Teknologi MARA (UiTM), Shah Alam, Selangor, \\ Malaysia, nanifadzlina@salam.uitm.edu.my \\ ${ }^{2}$ Mr., Fakulti Kejuruteraan Elektrik, Universiti Teknologi MARA (UiTM), Shah Alam, Selangor, \\ Malaysia, muhdfauzie93@gmail.com \\ ${ }^{3}$ Dr., Fakulti Kejuruteraan Elektrik, Universiti Teknologi MARA (UiTM), Shah Alam, Selangor, \\ Malaysia, suzis045@salam.uitm.edu.my \\ ${ }^{4}$ Dr., Fakulti Kejuruteraan Elektrik, Universiti Teknologi MARA (UiTM), Shah Alam, Selangor, \\ Malaysia, norsuzila@salam.uitm.edu.my \\ ${ }^{*}$ Corresponding Author
}

\begin{abstract}
This paper presents educational software based on MATLAB and a Graphical User Interface (GUI). This educational software consists of three subjects; electromagnetic, communication engineering and power engineering. MATLAB m-files are developed based on the requirements in the subjects. Then, its GUI are developed and integrated with the $\mathrm{m}$-files for ease of use. In this software, user is able to visualize the subjects from the plots, graphs, and images and obtain the answer automatically. User also does not need to install MATLAB in his/her Personal Computer (PC) because this software is developed with standalone program which allow user to use the software without installing Matlab. This software can be used by lecturers as teaching aid and self-learning materials among students, thus, help user to achieve better understanding on electrical engineering subjects. It is also found that this software has obtained positive feedback from lecturers and students as it attracts students in learning the subjects.
\end{abstract}

Keywords: MATLAB, Graphical User Interface (GUI), Electromagnetic, Communication Engineering, Power Engineering, Educational Tool.

\section{INTRODUCTION}

MATLAB is known as a Matrix Laboratory where it acts as a calculation software and high performance numerical analysis developed by Mathworks Company based in the USA. MATLAB has a unique advantage in form of digital signal processing, system identification, dynamic system simulation, graphic processing matrix algebra, numerical computing and many other areas [Lu Ling-yan et al.].

Furthermore, MATLAB GUI was used to design an interactive and visual educational tool developed for 
teaching and learning for signals and systems and digital signal processing [Han Ping et al.]. It was developed with five main function modules which is signal collecting, signal analysis, linear system analysis, filter design and signal processing techniques application module. This software designed a VHF (very high frequency) amplitude modulation signal analyzing and used with three kinds of analogue filters (Butterworth, Chebyshev, Ellipetic) and two kinds of digital filters (FIR DF and IIR DF). Besides that, this way includes with visual and graphic simulation which allow user to get better understanding.

The problem of dynamics of electric machines through simulation of an asynchronous machine can be solved by using MATLAB and GUI [Gabriela Dana Petropol-Serb et al.]. An asynchronous motor of a fan used in auxiliary's posts of electromotive was demonstrated. The software developed as a virtual laboratory where it can be a space when elements were expressed virtually by using interactive graphics and design. The result illustrated had shown the aim of their project which is to show the usage of GUI to build a typical process. The computational modeling of the induction machine had offers much information on the behavior and process control of the machine.

GUI (Graphical User Interface) and MATLAB are used to visualize EM fields of electromagnetic waves that are obtained by solving Maxwell's equations [A. Sinha et al.]. This method is used to solve the problem of resultant electromagnetic (EM) fields which is the combination of electric and magnetic fields where it complicated to visualize and understand. The aid of this GUI and MATLAB implemented is to develop understanding and designing of antenna and fading effects in wireless communication system. The implemented of GUI is used to observe and analyze the variation between electric field and magnetic field under different conditions.

Meanwhile, an educational tool was developed using the MATLAB Graphical User Interface (GUls) which are related with signal and systems [T. Mathumisaranon et al.]. The function of GUI module is to simulate the baseband of digital communication system. It will be demonstrated the transmission of the Binary Phase Shift Keying signal under an Additive White Gaussian Noise (AWGN) channel with an application of tone jamming interference and the reduction of the jamming effect using a digital notch filter at the part of receiver. The output response will be illustrated under the differences of the signal corresponding for each section of the communication system.

[Raymundo Cordero García et al.] has developed a simplified educational platform based on the integration of a Graphical User Interface (GUI) and FPGA for the didactic teaching of Space Vector Pulse Width Modulation (SVPWM). It is found that the platform helps in teaching and motivates research in SVPWM algorithms and FPGAs.

In addition, [Aleksei Tepljakov et al.] has designed a MATLAB with its graphical user interface (GUI) to show the time and frequency responses of FOS models and primary function of which is to learn fundamentals of fractional PID controller design.

In this project, educational software was developed using MATLAB and GUI which is flexible and user friendly. The scope of subjects that can be explored consists of 3 subjects; electromagnetic, communication engineering and power engineering which is suitable for electrical engineering undergraduates. The software was implemented with standalone program whereas user can use it without installing MATLAB software.

\section{METHODOLOGY}

The simulation tool used to develop this software is using MATLAB R2016a and its GUI. MATLAB allows an operation involved with manipulations of matrix, plotting of functions and data, implementation of algorithms, and interfacing with programs. Firstly, the information was gathered to choose a suitable equation that can be included in this software firstly. All the coding involved in this software were developed and saved in the form of m-files. Then, the GUI will be designed, and user can insert the input and then by clicking the push button an output or visualize the graph will appear. MATLAB program and GUI will be combined to create a system that can be used by user. Finally, a standalone program is developed so that this educational tool can be used without installing MATLAB software.

\subsection{Process of Developing M-File of Dot Product, Cross Product, Electric Field Intensity Due to Point Charge and Cylindrical Coordinate System}

Cross product can be obtained by multiplying the second term of each ratio by the first term of the other ratio in a proportion. Equation (1) shows the calculation of cross product which is related between dimension $\mathrm{x}, \mathrm{y}$, and $z$ of vector $a$ and $b$. 


$$
a \times b=\left|\begin{array}{ccc}
x & y & z \\
a_{1} & a_{2} & a_{3} \\
b_{1} & b_{2} & b_{3}
\end{array}\right|
$$

Meanwhile, dot product is an algebraic operation that takes two equal-length sequences of numbers (usually coordinate vectors) and returns a single number. Equation (2) shows the calculation of dot product as below.

$$
a \cdot b=\sum_{i=1}^{n} a_{i} b_{i}=a_{1} b_{1}+a_{2} b_{2}+\cdots+a_{n} b_{n}
$$

The electric field intensity $\vec{E}$ is defined as strength of an electric field at any point as stated in equation (3).

$$
\begin{gathered}
\vec{E}=\frac{k Q_{\text {source }}}{R^{2}}=\frac{Q_{\text {source }}\left(\overrightarrow{r_{2}}-\overrightarrow{r_{1}}\right)}{4 \pi \varepsilon_{0}\left|\overrightarrow{r_{2}}-\overrightarrow{r_{1}}\right|^{3}} \\
\vec{F}=\vec{E} Q_{2} \\
\vec{D}=\overrightarrow{\varepsilon_{0} E}
\end{gathered}
$$

Where,

$$
\begin{aligned}
& \mathrm{Q}=\text { Point Charge } \\
& \mathrm{R}=\text { Coordinate between two points of charge } \\
& \vec{E}=\text { Electric field intensity } \\
& \vec{D}=\text { Displacement of point charge } \\
& \vec{F}=\text { Force of electric field }
\end{aligned}
$$

Equations above are used to calculate the value of electric field intensity, E which involved two coordinates of point charge and source of point charge. While, the value of electric force, $F$ can be obtained when there is existence of another point charge, Q2. From equation (3), the value of the displacement can be determined which are related with a permittivity and electric field.

In coordinate system, there are three systems such as rectangular, cylindrical and spherical. $\mathrm{P}$ is represented as $(\rho, \varphi, z)$. The $z$ variable is similar with Rectangular coordinates. The ranges of the coordinate variables are available which $0 \leq \rho<\infty, 0 \leq \varphi<2 \pi$ and $-\infty<z<\infty$. A vector $A$ can be defined as

$$
\left(A_{\rho}, A_{\varphi}, A z\right) \text { or } A_{\rho} a_{\rho}+A_{\varphi} a_{\varphi}+A_{z} a_{z}
$$

Where the respective unit vectors lie on the $\rho$-, $\varphi$-, and $z$-directions. Hence, the relationships between Rectangular $(x, y, z)$ and Cylindrical $(\rho, \varphi, z)$ coordinates can be obtained as below.

$$
\begin{aligned}
& \rho=\sqrt{x^{2}+y^{2}}, \phi=\tan ^{-1}\left(\frac{y}{x}\right), z=z \\
& x=\rho \cos \phi, y=\rho \sin \phi, z=z
\end{aligned}
$$

\subsection{Process of Developing M-Files of Communication Engineering}

There are two types of PSK such as the Binary Phase-Shift Keying (BPSK) and Quadrature Phase-Shift Keying (QPSK) [Nani Fadzlina Naim et al.]. It has included the Frequency-Shit Keying (FSK) and AmplitudeShift Keying (ASK) under the topic of digital modulation. 


\subsubsection{Binary Phase-Shift Keying (BPSK)}

The Binary Phase-Shift Keying (BPSK) defined as two phases used and separated by $180^{\circ}$. BPSK is also known as Phase Reversal Keying or 2-PSK. The equation for BPSK is as below.

$$
s_{n}(t)=A \cos \left(2 \pi f_{c} t+\pi(1-n)\right), n=0,1
$$

where,

$\mathrm{Sn}(\mathrm{t})=$ sine integral of BPSK;

A = amplitude;

fc = carrier frequency;

$\mathrm{n}=$ phase shift of $180^{\circ}$.

\subsubsection{Quadrature Phase-Shift Keying (QPSK)}

Quadrature Phase Shift Keying (QPSK) is a form of Phase Shift Keying where the two bits are modulated at once and one of four possible carrier phase shifts $\left(0,90,180\right.$, or $\left.270^{\circ}\right)$ will be selected. The QPSK modulation can be calculated as below.

$$
s_{n}(t)=A \cos \left(2 \pi f_{c} t+(2 n-1) \frac{\pi}{4}\right), n=1,2,3,4
$$

Where,

$\mathrm{Sn}(\mathrm{t})=$ sine integral of BPSK;

$\mathrm{A}=$ amplitude;

fc = carrier frequency;

$\mathrm{n}=$ phase shift of $45^{\circ}$.

\subsubsection{Patch Antenna Analysis}

A patch antenna is also known as a rectangular micro strip antenna. This antenna has a lower profile in the radio antenna type because can be mounted with a flat surface [R. P. Meys et al.]. The value of $L$, length and $\mathrm{W}$, width have to configure before analyze the performance of patch antenna besides can visualize the $\mathrm{E}$ and $\mathrm{H}$ plane radiation patterns.

$$
W=\frac{c}{2 f o \sqrt{\frac{\left(\varepsilon_{r}+1\right)}{2}}}
$$

Where,

$\mathrm{W}=$ width of patch;

$c=$ speed of light $\left(3 \times 10^{8}\right)$;

fo = resonance frequency;

$\varepsilon \mathrm{r}=$ dielectric constant.

$$
L=L e f f-2 \Delta L
$$

Where,

$\mathrm{L}=$ sine integral of BPSK;

Leff = effective length of patch;

$\Delta \mathrm{L}=$ length extension of patch . 


$$
\begin{array}{r}
(E \phi)_{E}=\frac{\sin \left(\frac{K_{0} h}{2} \cos \phi\right)}{\frac{K_{0} h}{2} \cos \phi} \cos \left(\frac{K_{0} L}{2} \sin \phi\right) \\
(E \phi)_{H}=\sin \theta \frac{\sin \left(\frac{K_{0} h}{2} \cos \phi\right)}{\frac{K_{0} h}{2} \cos \phi} \frac{\sin \left(\frac{K_{0} W}{2} \cos \theta\right)}{\frac{K_{0} h}{2} \cos \theta}
\end{array}
$$

Where,

$$
\begin{aligned}
& (E \phi) E=E-p l a n e ; \\
& (E \phi) H=H \text {-plane; } \\
& K 0=\text { free space wavenumber; } \\
& h=\text { thickness of substrate. }
\end{aligned}
$$

The equations above are part of the calculation involved in patch antenna analysis. W was represented as width of patch while $L$ was represented as length of patch. From that, the value of $E$-plane and $\mathrm{H}$-plane can be obtained.

\subsection{Process of Developing M-Files of Power Engineering}

The 3-phase resistive system is related which allow user to explore about the conversion of star and delta either delta to star or star to delta that connected with the load (resistor) [W. Al-Khateeb et al.].

\subsubsection{Delta to Star Resistive Conversion}

The conversion from delta to star can be seen as the following in Fig. 1.

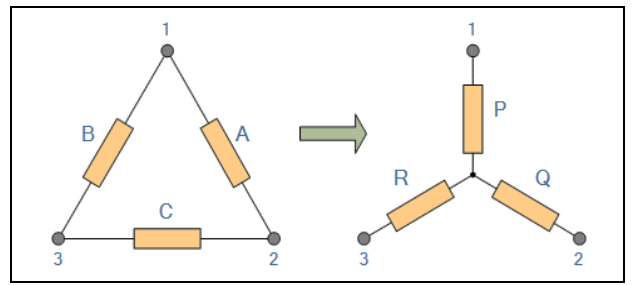

Fig. 1. Delta to Star conversion.

The calculation of delta to star transformations is as shown below.

$$
\begin{aligned}
& P=\frac{A \times B}{A+B+C} \\
& Q=\frac{A \times C}{A+B+C} \\
& R=\frac{B \times C}{A+B+C}
\end{aligned}
$$

\subsubsection{Star to Delta Resistive Conversion.}

In Fig. 3 shows a transformation of resistive from Star to Delta.

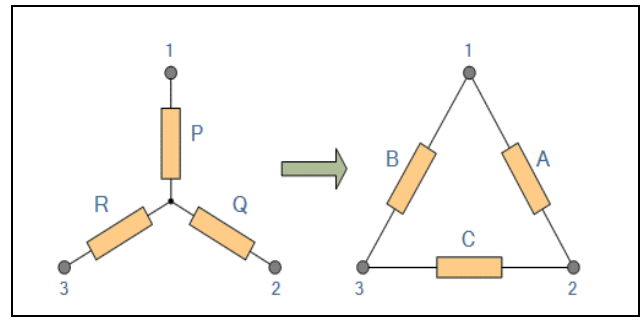

Fig. 3: Star to delta conversion. 
The calculations of conversion referred as below:

$$
\begin{aligned}
& A=\frac{P \times Q}{R}+Q+P \\
& B=\frac{R \times P}{Q}+P+R \\
& C=\frac{Q \times R}{P}+Q+R
\end{aligned}
$$

\subsection{Software Development}

The software used in this project is MATLAB R2016a with its Graphical User Interface (GUI) or Graphical User Interface Design Environment (GUIDE) where it provides point-and-click control of software applications. The GUI typically contains controls such as menus, toolbars, buttons, and sliders. The advantage of using this is that it does not require user to learn a language or type commands in order to run the application. In order to develop this educational tool, a programmer needs to design MATLAB program using $\mathrm{m}$-files based on the problem of the subjects. Then, the $\mathrm{m}$-files will be integrated with its GUI. Lastly, the overall standalone system will be developed. The use of the GUIDE Layout Editor enables the programmer to design the layout of the system easily. The GUIDE will then generate the $\mathrm{m}$-files automatically.

\section{RESULT AND DISCUSSION}

The system starts with Main menu as shown in Fig. 4 which consists of three options subject; electromagnetic, communication engineering and power engineering. The 'Help' button is provided to aid user with the description of the program. For the first subject is Electromagnetic where it consists of six subtopics that user can explore which is electric field intensity, electric field plot, cross and dot product, electromagnetic propagation and coordinate system. The second subject involved is Communication Engineering where it consists of three subtopics such as digital modulation, high frequency analysis and patch antenna analysis. For Power Engineering, there are two subtopics that user can choose either star to delta conversion or delta to star conversion.

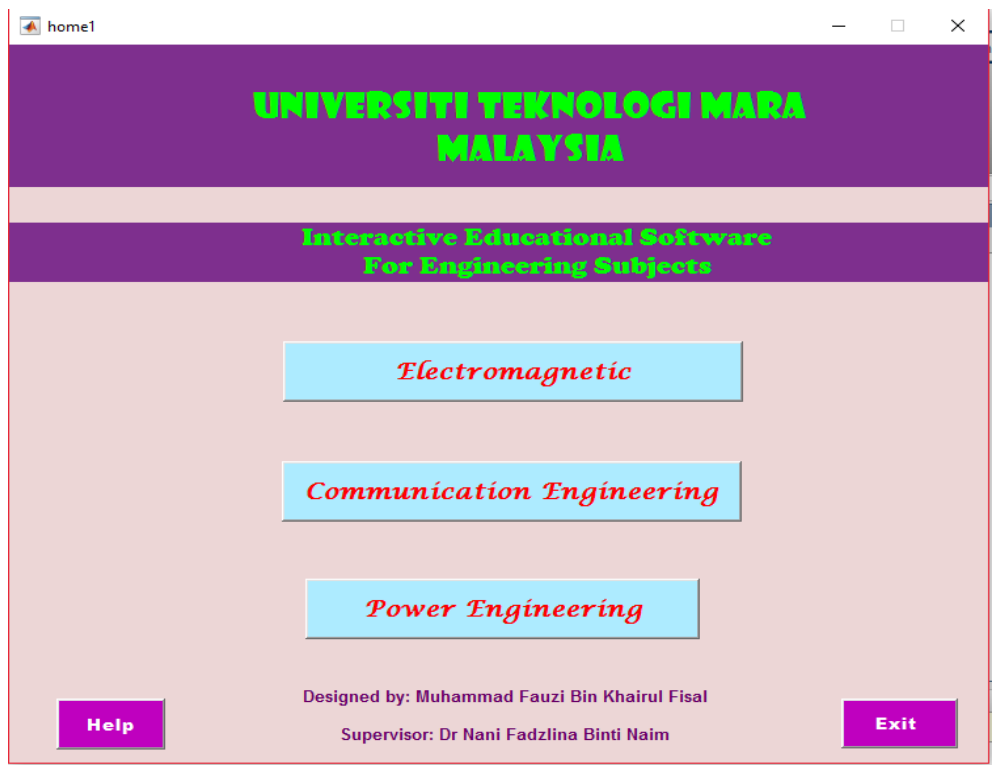

Fig. 4. Main menu of educational software

For electromagnetic, user has to enter the desired value of vector $P$ and $Q$ according to the dimensions $x, y$ and $z$.The 'Answer' button is provided to show the answer for Dot Product when it is clicked as shown in Fig. 5 . 


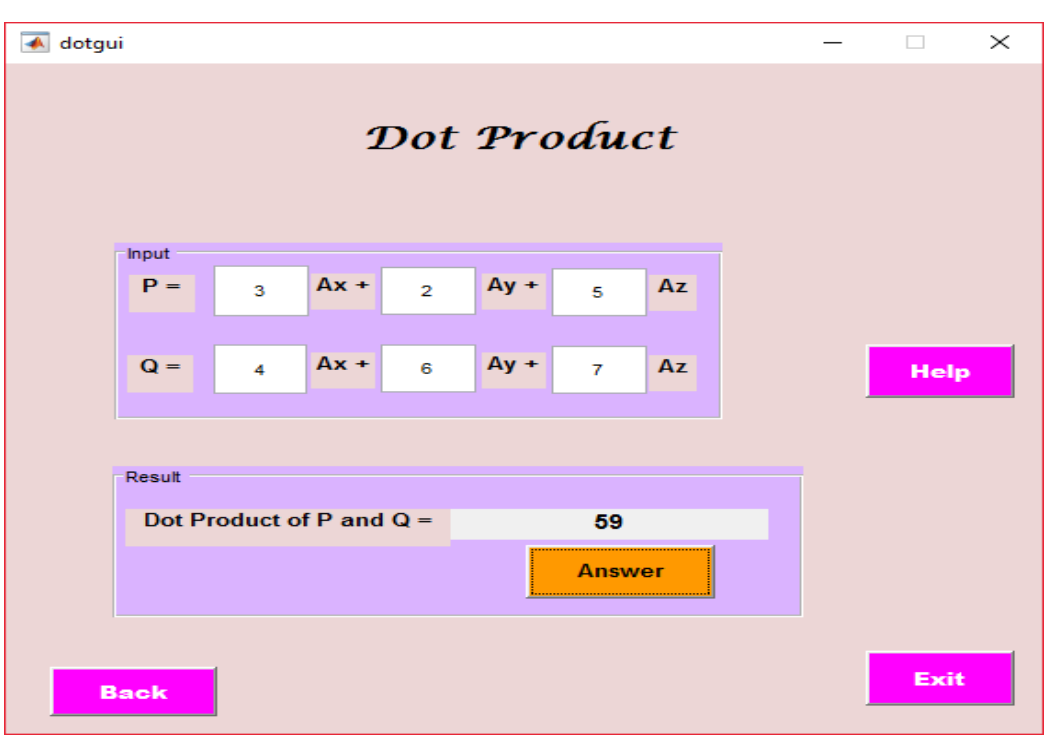

Fig. 5. Dot Product calculator

Based on Fig. 6, user is required to insert the coordinate of the charge and the observation point. The source point is labeled as $Q$ where it consists of the charge value and the coordinate of the point. Meanwhile, point of observation is labeled as $P$. The electric force and the displacement of point charge can be obtained by inserting the second point of charge. The answer can be obtained when user inserted all the parameter required.

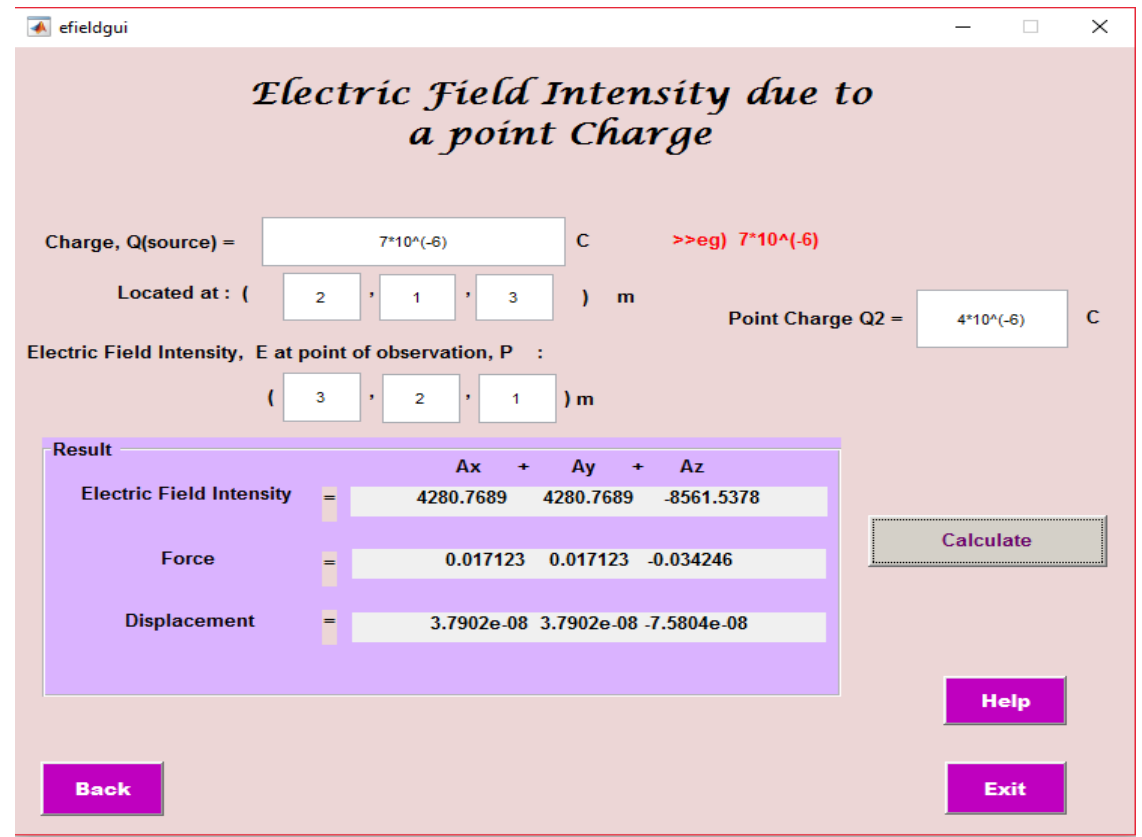

Fig. 6. Electric Intensity Field due to a point charge

Based on Fig. 7, cylindrical coordinate conversion is subtopics of coordinate system that are provided in Electromagnetic topic. In this subtopic, user inserted the value of rho, phi and $z$ to convert in rectangular and spherical coordinate. All the answers are shown in below after 'Calculate' button generated by the user. 


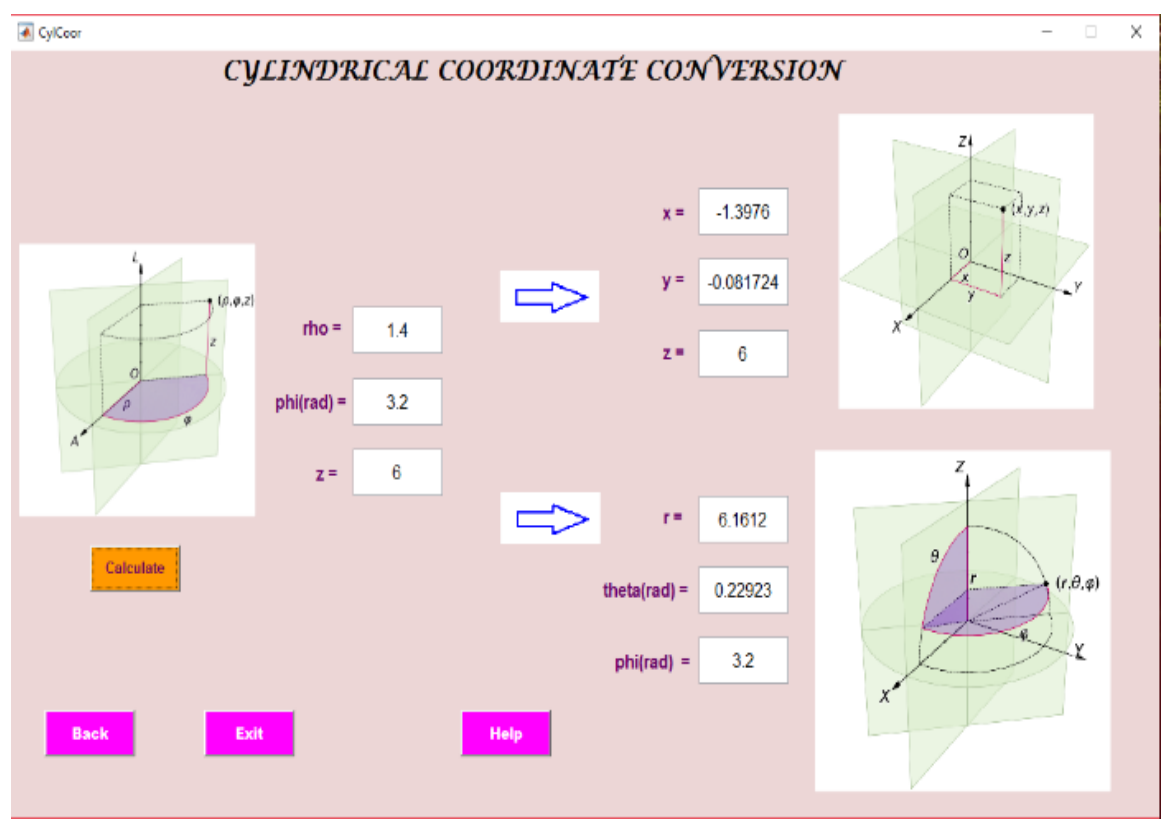

Fig. 7. Cylindrical coordinate conversion

The other topic is provided in this software is the wave propagation of electromagnetic. User can be observed the propagation of electromagnetic wave under two different conditions; free space and good conductor. The wave propagation of electromagnetic will be generated when user clicking a pushbutton and it does not need an input value to be inserted. Based on Fig. 8, the wave propagation of electromagnetic result has shown under free space condition and good conductor condition.

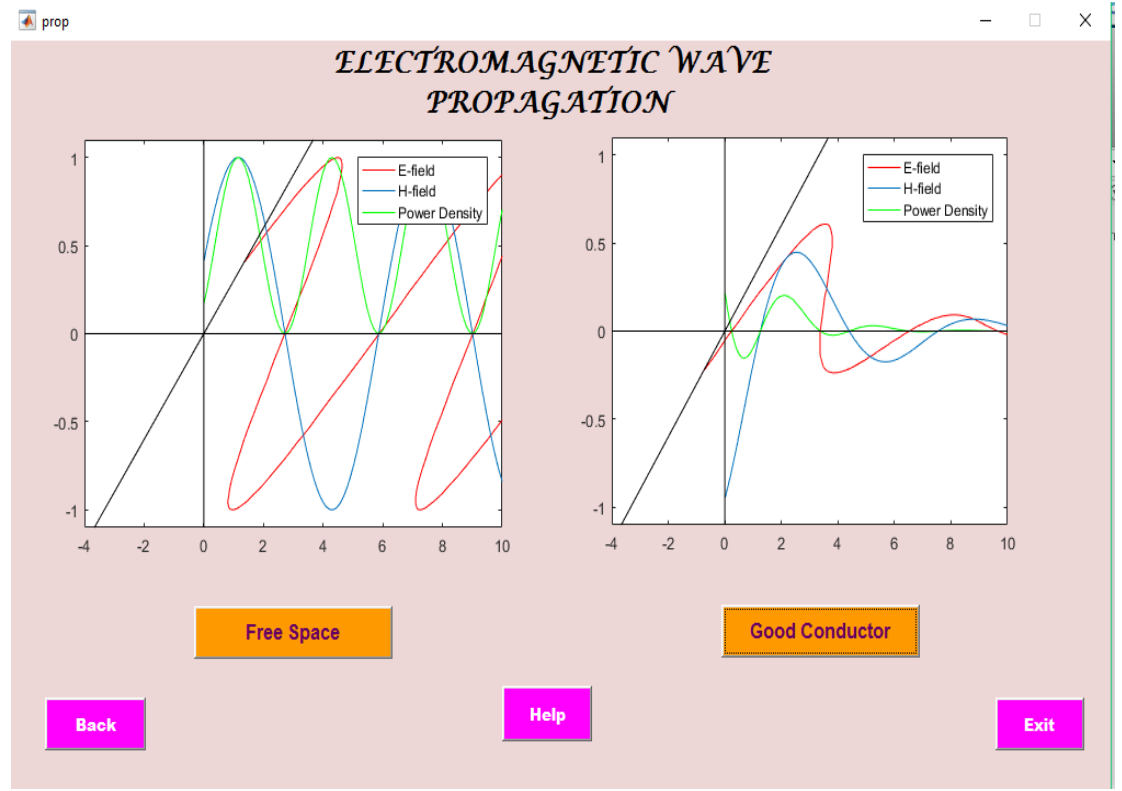

Fig. 8. Electromagnetic wave propagation

In this part, user is required to insert suitable values of carrier of frequency and binary input signal which is low signal frequency, high signal frequency, and binary input of the signal. By clicking the 'Generate!' button, the signal will be plotted at the signal output of modulation. The combination signals of four digital modulations can be obtained as shown in Fig. 9. 


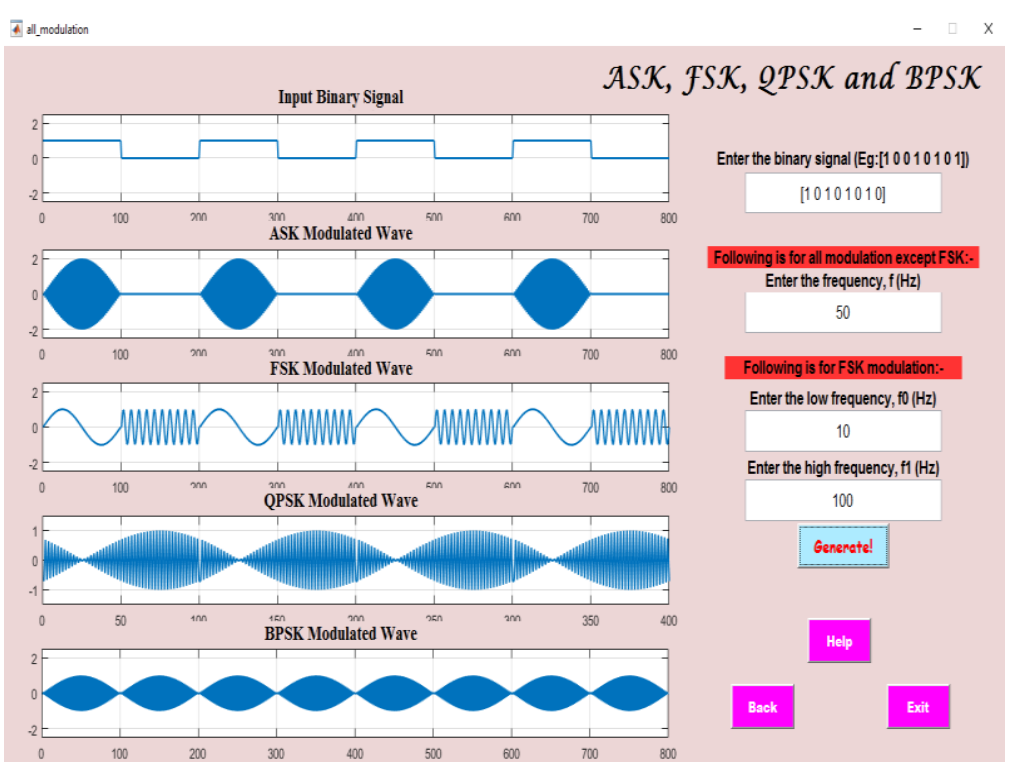

Fig. 9. Signal Modulation analysis

Fig. 10 is to analyze the performance of patch antenna. User has to insert the desired values; carrier frequency, dielectric constant and the thickness of patch in appropriate input box. The value of frequency, dielectric constant and thickness of patch inserted which is equal to $2.3 \mathrm{~Hz}, 3.38$ and $30 \mathrm{~mm}$; respectively. The result obtained after clicking the 'Calculate 'button is shown below. The radiation pattern of $\mathrm{E}$ and $\mathrm{H}$ planes will be plotted based on the desired input. The result for the dimension of patch antenna can be obtained in term of length and width in millimeter units. Other than that, this calculator can calculate efficiency and gain of patch antenna.

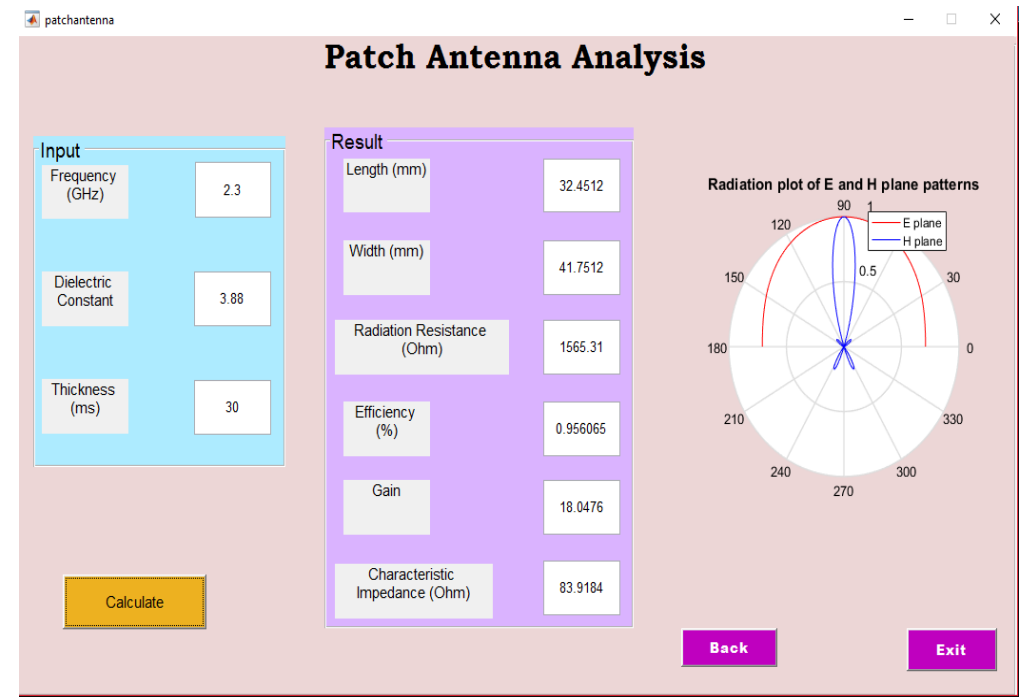

Fig. 10. Patch antenna analysis calculator

For Power Engineering, 'Star to Delta' or 'Delta to Star' conversions are provided for user to choose for conversion. User must insert an appropriate value and then click 'Calculate' button to see the result or answer. Fig. 11 is the result of calculating the 'Star to Delta' conversion when user inserts the values of $X, Y$ and $Z$. 


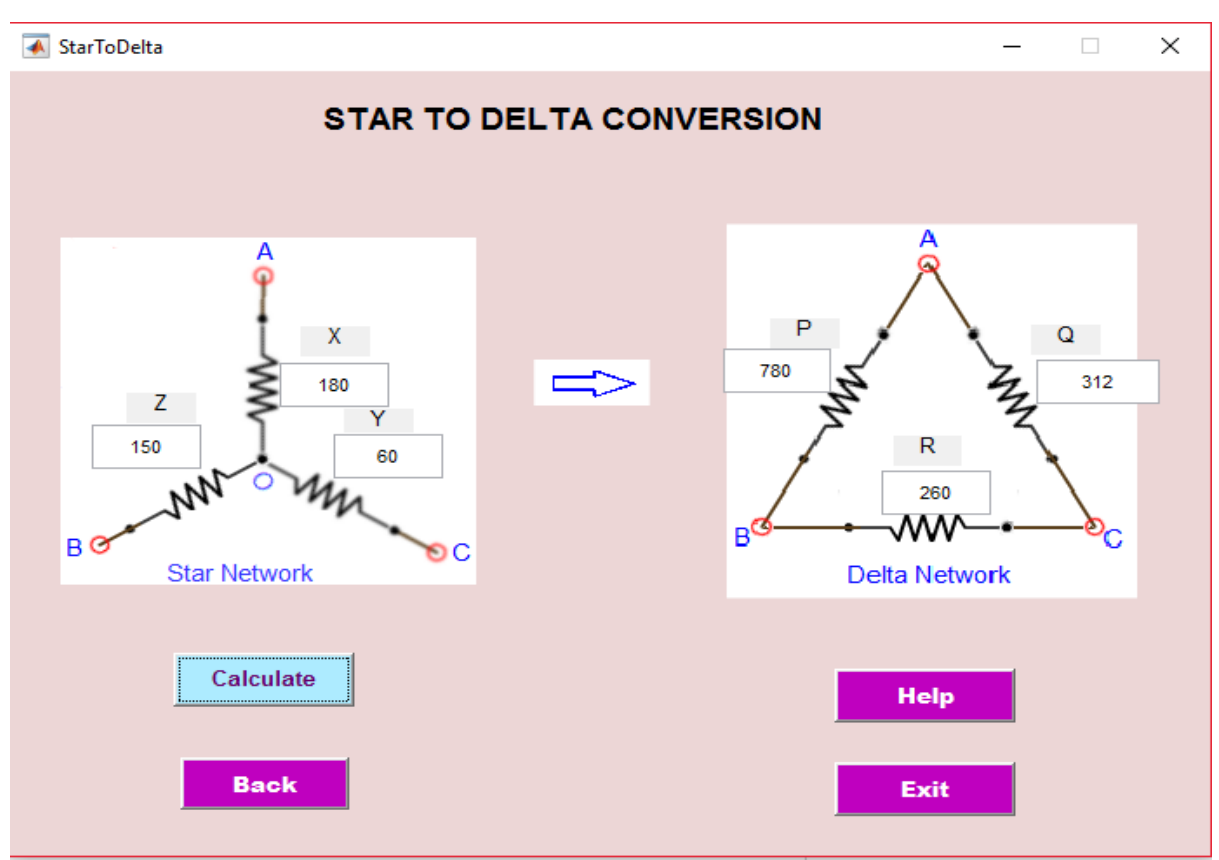

Fig. 11. Star to Delta conversion calculator

Based on Fig. 12, it displayed Delta to Star conversion for Power Engineering topic. This conversion is to convert a resistive load in form of delta to the form of star. Fig. 12 below shows the answer of conversion after the user inserts the appropriate input values and clicking the 'Calculate' button.

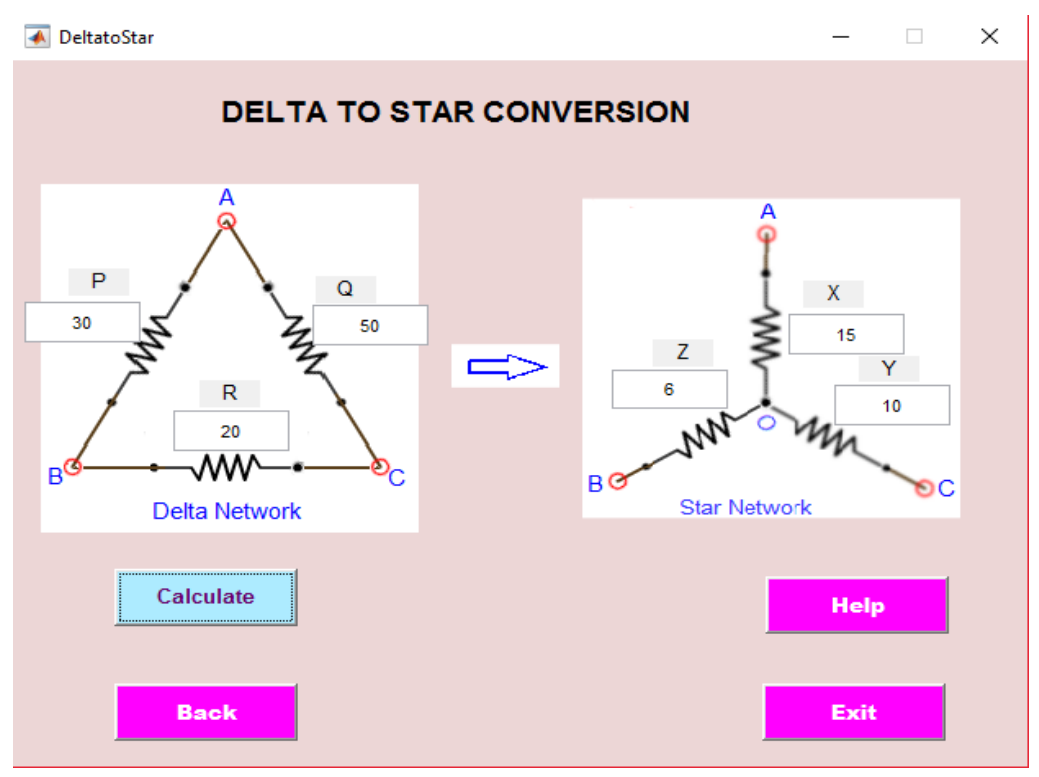

Fig. 12. Delta to Star conversion calculator

\section{CONCLUSION}

In conclusion, an educational tool using MATLAB and its GUI has been developed which gives benefit to the user either in learning or teaching purposes with three subjects of electrical engineering involved. The system established for the students to reaffirm their understanding in the three different subjects of electrical engineering such as electromagnetics, communication engineering and power engineering. Besides, standalone program of this educational tool also has been developed. Therefore, user does not need to install MATLAB in order to use this educational software. For future work, this software can be expanding by adding more scopes that are related with electrical engineering subjects. 


\section{ACKNOWLEDGMENT}

This paper is part of research work that is supported by Faculty of Electrical Engineering, Universiti Teknologi MARA (UiTM) Shah Alam, Selangor, Malaysia.

\section{REFERENCE LIST}

Lu Ling-yan and Yao Jin-li, Design and Implementation of Linear Control System Teaching Software Using MATLAB-based GUI and Microcontroller, IEEE Journal, 2009.

Han Ping, He Weikun, Shi Qingyan and Han Yan, "An educational tool design for the course of signal processing based on Matlab GUI," 2014 IEEE International Conference on Teaching, Assessment and Learning for Engineering (TALE), Wellington, 2014, pp. 131-134.

Gabriela Dana Petropol-Serb, Ion Petropol-Serb, Aurel Campeanu and Anca Petrisor, Using GUI of MATLAB to Create a Virtual Laboratory to Study an Induction Machine, IEEE Journal, 2007.

A. Sinha, C. Mandal and S. K. Mandal, "Implementation of a GUI to visualize EM fields using MATLAB," 2016 International Conference on Microelectronics, Computing and Communications (MicroCom), Durgapur, 2016, pp. 1-6.

T. Mathumisaranon and P. Chayratsami, "MATLAB GUI for digital communication system with tone jamming," Proceedings of 2013 IEEE International Conference on Teaching, Assessment and Learning for Engineering (TALE), Bali, 2013, pp. 589-592.

Nani Fadzlina Naim, Abdul Hadi Nordin and Husna Ab-Rahman, Interactive Learning Software for Communication Engineering Subjects, IEEE Journal, 2011.

R. P. Meys and A. Rouibah, "Six Easy Steps That Explain the Radiation of the Rectangular Patch Antenna [Education Corner]," in IEEE Antennas and Propagation Magazine, vol. 58, no. 6, pp. 95-101, Dec. 2016.

W. Al-Khateeb, S. Al-Irhayim and K. Al-Khateeb, "Reliability evaluation of scalable complex networks through delta-star conversion," 2008 International Conference on Computer and Communication Engineering, Kuala Lumpur, 2008, pp. 1162-1166.

Raymundo Cordero García, Igor Esdras Silva Ono, Vitória dos Santos Fahed, João Onofre Pereira Pinto, "Simplified educational platform for SVPWM control of a two-level three-phase inverter using Matlab GUI and FPGA," 2017 Brazilian Power Electronics Conference (COBEP), pp. 1-6.

Aleksei Tepljakov; Eduard Petlenkov; Emmanuel A. Gonzalez; Ivo Petras, "Design of a MATLAB-based Teaching Tool in Introductory Fractional-Order Systems and Controls," 2017 IEEE Frontiers in Education Conference (FIE), pp. 1-5. 